Le genre de la maladie : pratiques, discours, textes et représentations

\title{
À propos de la maladie dans l'œuvre de Shi Pingmei (1902-1928)
}

Jacqueline ESTRAN

\section{(2) OpenEdition \\ Journals}

Electronic version

URL: https://journals.openedition.org/transtexts/660

DOI: $10.4000 /$ transtexts. 660

ISSN: 2105-2549

Publisher

Gregory B. Lee

Electronic reference

Jacqueline ESTRAN, "À propos de la maladie dans l'œuvre de Shi Pingmei (1902-1928)", Transtext(e)s Transcultures 跨文本跨文化 [Online], 11 | 2016, Online since 30 June 2017, connection on 21

September 2021. URL: http://journals.openedition.org/transtexts/660 ; DOI: https://doi.org/10.4000/ transtexts.660

This text was automatically generated on 21 September 2021.

(c) Tous droits réservés 


\title{
À propos de la maladie dans l'œuvre de Shi Pingmei (1902-1928)
}

\author{
Jacqueline ESTRAN
}

\section{Le genre de la maladie en Chine}

1 En tant que matérialisation - support d'une identité, le corps sert de point de départ à un travail sur la représentation qui fait intervenir tout un ensemble de disciplines et de paradigmes, décliné en fonction d'approches spécifiques. ${ }^{1}$ C'est, en général, essentiellement dans sa dimension souffrante qu'il est appréhendé, une souffrance qui fait jouer la question du genre et se conjugue de façon différente au masculin ou au féminin.

2 Les rapports entre maladie et femmes ont donné naissance à un genre de la maladie qui s'est constitué, en Chine, sur plus de 2000 ans. Sur un plan extradiégétique, ils concernent la manière dont les maladies touchant les femmes sont abordées sur un plan culturel et médical et, sur un plan intradiégétique, la manière dont les femmes ont géré ce rapport à la maladie.

3 Pour ce qui est du statut culturel de la maladie, il existe, en Chine, depuis le III siècle de notre ère une "médecine des femmes ", qui s'est surtout développée et institutionnalisée à partir du $\mathrm{X}^{\mathrm{e}}$ siècle. Contrairement à ce qui s'est passé en Occident dans le domaine médical, la construction de la différence ne s'est pas faite en Chine uniquement sur une différence biologique, elle s'est appuyée sur les principes du Yin et du Yang, principes masculin et féminin fondateurs d'une conception du monde, qui ont coexisté avec le sentiment de leur nécessaire complémentarité. Cette conception du monde, basée sur une partition entre deux principes complémentaires, a nécessairement joué un rôle dans la mise en place et le statut de cette médecine des femmes puisque ces principes sont également à la base de la médecine chinoise, et que c'est de leur équilibre que dépend la santé. ${ }^{2}$ Mais ils n'échappent pas pour autant à la hiérarchie, une hiérarchie déterminée par la tradition confucéenne régissant l'ordre social, selon laquelle les individus se 
définissent par leurs relations, la femme se devant d'être soumise à son père, puis à son mari, puis à son fils. ${ }^{3}$

$4 \mathrm{Si}$, jusqu'au XIV e siècle, les femmes ayant la possibilité d'écrire sont peu nombreuses, comparativement aux hommes, la situation évolue alors et elles s'expriment de plus en plus, tant dans les genres mineurs et peu valorisés (prose, fiction) que dans le domaine de la poésie. Cette évolution atteint son apogée au XVII siècle : non seulement, à la faveur d'un changement dynastique ayant entraîné un état de guerre prolongé, elles témoignent des souffrances générées par la guerre et font entendre leurs revendications mais elles agissent, prenant leur place dans la société et, parfois, les armes. ${ }^{4}$ Mais si la crise sociale et politique ouvre une fenêtre de liberté, celle-ci est rapidement refermée par un ordre traditionnel encore trop puissant pour laisser la parole aux femmes et c'est alors que commence à circuler l'expression qui veut que la «vertu d'une femme est d'être sans talent $\|^{5}$, position qui prendra un pouvoir tel que jusqu'au début du XXe siècle, il sera mal vu d'avoir une fille talentueuse, sur un plan intellectuel, et celles-ci seront souvent empêchées par leur propre famille d'étudier.

Dans ce contexte où naît et s'affirme la volonté de s'exprimer, c'est la maladie qui, d'une certaine façon, sert les femmes, comme l'analyse Grace Fong: sous les Ming et les Qing $\left(\mathrm{du} \mathrm{XIV}^{\mathrm{e}}\right.$ siècle au début $\left.\mathrm{du} \mathrm{XX}^{\mathrm{e}}\right)$ convalescentes, elles sont dispensées des tâches quotidiennes qui les accaparent d'ordinaire, et peuvent, grâce à la maladie, bénéficier d'un espace, d'une " chambre à elles ", pour écrire et échapper à une vie sociale souvent bien éloignée de leurs idéaux. Une véritable littérature féminine s'est alors développée et les contraintes d'une santé fragile se sont accompagnées du plaisir d'une liberté retrouvée, une liberté consistant en du temps pour soi. ${ }^{6}$ Cette évocation de la maladie ou du corps malade dans les écrits féminins est à mettre en rapport avec le bandage des pieds, les dynasties Ming et Qing correspondant à la période où cette pratique (apparue au Xe siècle) s'est abondamment répandue. Malgré ce qu'elle avait de douloureux et de handicapant, elle était, du fait de son institutionnalisation, incontournable, et a amené les femmes à chercher ailleurs, hors de ce corps meurtri, une maîtrise qu'elles avaient perdue sur le plan physique. ${ }^{7}$

6 Dès lors, statut et représentation de la maladie se sont inscrits dans une histoire des femmes et du genre qui leur donne sens et légitime leur interrogation à l'orée de cette ère nouvelle que représente le début du XXe siècle et plus particulièrement les années 1920, une période qui se caractérise par l'éclatement de tous les repères, en particulier pour les femmes, et qui sera questionnée au travers de l'œuvre de Shi Pingmei石评梅 (1902-1928).

\section{Shi Pingmei}

7 Shi Pingmei, jeune écrivaine brillante au destin singulièrement bref, appartient à la première génération d'écrivaines de ce qu'il est convenu d'appeler la «nouvelle littérature » en Chine, la littérature en langue vernaculaire (baihua) qui s'impose à partir de 1918 et marque une rupture avec la tradition littéraire et la langue classique, le wenyan . Malgré sa courte existence (elle décède à 26 ans), Shi Pingmei est considérée comme une écrivaine majeure de la première moitié $\mathrm{du} \mathrm{XX}^{\mathrm{e}}$ siècle ${ }^{8}$. Elle s'est beaucoup impliquée dans la vie littéraire de la Chine des années 1920, par ses poèmes, mais également dans le combat pour l'émancipation des femmes, par ses essais sur les droits des femmes, le marxisme et les réformes sociales. Elle a également une activité éditoriale, et cela dès 
l'adolescence, qu'elle poursuivra jusqu'à son décès, publiant différents périodiques alliant son intérêt pour la littérature à son engagement social. ${ }^{9}$

8 Elle est, par ailleurs, représentative de la petite communauté de femmes / intellectuelles qui s'est constituée au tout début des années 1920 au sein de l'École normale supérieure pour filles de Pékin, une communauté qui constitue un repère au sens positif, un lieu où ces étudiantes se retrouvent libres et indépendantes, juste elles-mêmes. De la chambre que Shi Pingmei partage avec Lu Yin à l'École normale à celle qu'elle occupe en tant qu'enseignante des classes préparatoires de cette même école, et qui prendra pour ses amies le nom de nid, ${ }^{10}$ c'est un lieu physique mais c'est aussi et surtout un espace qui échappe aux normes sociales et dans lequel elles construisent, en s'appuyant sur l'écriture, leurs propres normes, visions du monde et façons d'être. Cette communauté, Shi Pingmei la déploie bien au-delà de son cercle d'amies, créant une véritable communauté de lectrices, ainsi que l'analyse Jocelyn Zelpha Chambers dans la thèse qu'elle a consacrée à l'écrivaine. ${ }^{11}$

9 Si ce sont Shi Pingmei et son œuvre qui servent ici de fil conducteur, elle ne peut être isolée de ses amies, elles aussi écrivaines, en particulier Lu Jingqing 陆晶清 (1907-1993) et Lu Yin 庐隐 (1898-1934), elles-mêmes touchées par diverses maladies et dont l'histoire recoupe celle de Shi Pingmei en de nombreux points. Lu Yin retrace notamment la courte existence de Shi Pingmei, dans un roman publié en 1934: Xiangya jiezhi 象牙戒指 [Anneaux d'ivoire, 1934] tandis que Lu Jingqing échange avec elle de nombreux poèmes, qu'elles publient, contribuant à la mise en place de la communauté de lectrices qui se forme autour de Shi.

\section{Shi Pingmei, écriture et maladie}

10 La représentation du corps en Chine s'inscrit dans une longue tradition d'idéalisation - ou de non réalisme - qui a favorisé la conception du corps comme « signe renvoyant à autre chose que lui-même " pour reprendre l'expression de Cécile Toublet. ${ }^{12}$ La maladie - qui touche le corps - représente donc d'emblée autre chose qu'une simple affection du corps.

En préalable, il me semble important de distinguer la maladie et son champ sémantique de celui de la mort et du désespoir, deux registres qui sont assez souvent associés dans les études sur l'œuvre de Shi Pingmei mais qui relèvent de deux dynamiques différentes quelle que soit leur complémentarité -, l'une tournée vers la vie, l'autre vers la mort, et sont symptomatiques des tensions qui ont traversé l'existence de Shi.

Le corpus retenu pour aborder ce rapport de Shi Pingmei à la maladie est celui de ses nouvelles, de la première écrite en 1923 à son décès en 1928, soit 14 textes. Si le caractère autobiographique de ces nouvelles est indéniable, le choix de les revendiquer comme fiction implique une mise à distance qu'il convient de prendre en compte dans la mesure où Shi Pingmei a écrit et publié de nombreux textes directement autobiographiques (poèmes, proses variées, littérature épistolaire).

13 Avec la première nouvelle qu'elle publie sous le titre de Bing 病 [Maladie, 1923], Shi Pingmei inscrit d'emblée son écriture dans cet espace de la maladie qui s'est, au cours des siècles précédents, défini comme proprement féminin. Mais que représente la «maladie » pour Shi Pingmei, à ce moment? 

particulier, une situation de crise qui affecte Shi Pingmei sur un plan personnel et dans sa créativité. En effet, peu de temps après son arrivée à Pékin où elle n'a pas de famille (elle est originaire du Shanxi et a étudié à Taiyuan avant d'intégrer l'Éole normale supérieure de Pékin), elle est abusée par un homme, une relation de son père à qui il l'avait recommandée et qui était censée la protéger dans cette vaste ville. Cette première expérience qui la confronte d'abord à l'illusion de l'amour puis à la trahison l'affecte profondément. ${ }^{15}$

18 Elle vit alors aussi un premier échec dans le monde littéraire. En 1922, elle a publié une pièce de théâtre qui lui a valu de nombreux problèmes: elle y présentait un couple d'étudiants chinois qui, s'étant rencontré aux États-Unis, se sépare une fois de retour en Chine, l'homme se laissant convaincre par son père d'accepter le mariage arrangé que celui-ci lui a préparé. La jeune femme, elle, non seulement n'accepte pas, mais elle empoisonne celle qu'elle identifie comme sa rivale. La pièce a fait scandale et Shi Pingmei a dû se justifier quant à ce dénouement pour le moins inattendu. ${ }^{16} \mathrm{En}$ général, dans ce 
genre de situation, à l'époque, les amants se suicident ensemble ou alors la femme meurt de désespoir ou se suicide. La vengeance de la femme délaissée est rare et totalement inédite sous la plume d'une jeune femme inconnue de 20 ans. Si cette vengeance peut apparaître comme un exutoire à la trahison vécue par Shi Pingmei dans sa relation avec l'ami de son père, elle montre surtout, d'une part, que l'écriture lui permet de transgresser des tabous, et, d'autre part, qu'elle a pour volonté d'explorer les méandres de l'âme humaine, avec originalité, à partir de son expérience personnelle.

Dans la nouvelle « Bing », la narratrice passe assez rapidement de la maladie physique à la souffrance émotionnelle, une souffrance dont elle a conscience que ce n'est pas dans son corps que s'en situe l'origine: "Je vois mon sang tomber, goutte à goutte, de mon cœur dans la terre; mes larmes couler, une à une, de mes yeux dans mon cœur; en un an, tout ce que j'ai obtenu, c'est une douleur infinie... $»^{17}$. C'est le jour de l'an, la période de l'année où la réunion familiale est quasi incontournable en Chine, et la narratrice pense à sa famille qui est au loin, se remémorant un autre jour de l'an où, enfant, elle était aussi malade mais pouvait alors bénéficier de l'attention et de la tendresse de sa mère et de son père.

Cette maladie donne à la narratrice conscience de ses limites et de sa faiblesse mais aussi de la relativité de la souffrance : «Je suis enfermée dans la prison de la maladie mais, ami, regarde ce monde du haut de ton nuage, qui ne souffre pas? $»^{18}$.

21 La maladie représente aussi une pause, au cours de laquelle la narratrice apprend à reconnaître et à se tourner vers ce qui lui apporte du réconfort : la nature et la pensée de sa mère. Progressivement, la narratrice prend conscience de la possibilité qu'elle a de lutter et d'aller au-delà du moment présent, aussi douloureux soit-il.

Shi Pingmei s'inscrit avec ce texte dans un espace de création féminin double pourrait-on dire,

23 -par la maladie - une maladie qui, certes, affaiblit mais ouvre aussi une porte sur un espace hors du quotidien et lui permet de rejoindre la cohorte de ses sœurs des siècles précédents, sœurs majoritairement poétesses, qu'elle ne pouvait ignorer écrivant ellemême de la poésie,

-par la référence à sa mère, filiation qu'elle se réapproprie d'une certaine façon, puisque si c'est sa mère qui lui a donné la vie, c'est à présent elle-même qui se la redonne en dépassant la maladie et la donne à sa mère au travers du combat qu'elle mène contre la maladie ;

Adoubée d'une certaine façon par cette maladie qui, en l'inscrivant dans une double filiation -littéraire féminine et maternelle - lui donne accès à l'écriture, elle va pouvoir partir en exploration. Et effectivement, c'est après l'écriture de ce texte qu'elle obtient la reconnaissance du monde littéraire pour ses premiers poèmes publiés et se lance dans une pratique de l'écriture qui l'accompagnera toute son existence (même si elle ne s'essaie plus au genre dramatique).

Si la narratrice de la nouvelle est malade, qu'en est-il de l'auteure, Shi Pingmei ? Lu Yin, dans son roman, dit que personne ne sait où elle est, puis qu'elle réapparaît, disant que tout va bien désormais. De sa maladie, on ne sait, comme dans sa nouvelle, quasiment rien, si ce n'est qu'elle a commencé comme un rhume. Lu Yin l'interroge afin de pouvoir compléter le journal qu'elle écrit, mais sans résultat. ${ }^{19}$ 


\section{De malade à soignante}

Après cette nouvelle, Shi Pingmei se livre à une écriture plus directement autobiographique tournée vers la communauté de femmes qui l'entoure (et au-delà) tout en traversant des épreuves qui l'ont beaucoup affectée - parmi lesquelles, une impossible histoire d'amour qui se termine par le décès de l'homme aimé. ${ }^{20}$ Elle publie deux nouvelles en 1925 puis une dizaine en 1927-28. Elle y explore le devenir des femmes de son époque sous différentes facettes : jeunes femmes intellectuelles ou artistes en butte à la mentalité traditionnelle, jeunes femmes délaissées par leur mari, femmes célibataires, jeunes femmes engagées dans un combat révolutionnaire.

La maladie, qui y sert de fil conducteur, témoigne d'une évolution: elle passe progressivement de la figure de malade à celle de soignante.

Outre la nouvelle "Bing", on retrouve la figure de la jeune femme malade dans "Liulangde gezhe » (1927) [Le chanteur errant]. ${ }^{21}$ Celle-ci guérit rapidement grâce à un séjour en bord de mer avec son père, dans la maison du grand-père, alors que la mère est décédée et que le père la pleure. Artiste, c'est la beauté de la nature qui lui permet de se remettre alors que, dans le même temps, elle est confrontée au désespoir d'un musicien errant, auquel elle apporte un peu de réconfort mais qui, ayant tout perdu (famille massacrée) fait le choix de se suicider. Il laisse à la jeune femme une lettre dans laquelle il lui confie son histoire, en faisant le témoin des drames de leur époque. Consciemment ou pas, avec ce texte, Shi Pingmei endosse le rôle de celles qui l'ont précédée, sous les Ming et les Qing, en conservant le souvenir du drame des êtres humains et en le transmettant. ${ }^{22}$ Ensuite, lorsque la maladie est évoquée, c'est au travers de la figure de l'infirmière, figure donc de soignante et non plus de malade, avec des variantes qui témoignent des préoccupations de Shi Pingmei à l'époque.

31 Le doute et la recherche de soi sont associés à cette figure d'infirmière et cela sur de multiples plans: Chine et Occident, modernité de la jeune femme active professionnellement et tradition des origines. Dans « Daogao - Wanwan de riji » (1927) [Prière - Journal de Wanwan] ${ }^{23}$, Shi Pingmei présente l'histoire d'une jeune femme, orpheline, qui, recueillie par une missionnaire, apprend le métier d'infirmière et l'exerce ensuite dans un hôpital sous les ordres d'un médecin. Confrontée à un jeune homme, amené par ses collègues dans un état critique suite à un chagrin d'amour, la jeune infirmière se prend d'amitié pour lui au point d'imaginer qu'il est peut-être le frère qu'elle aurait aimé avoir, la famille qui lui manque tant. Mais il meurt avant qu'elle ait la possibilité d'évoquer cela avec lui et personne ne venant réclamer ses effets personnels, elle conserve son journal, héritière finalement de la mémoire de cet homme.

Si la fonction première de cette figure est de soigner, elle apparait aussi comme une jeune femme en mal de reconnaissance, d'identité et elle ne peut soigner le jeune homme qui a renoncé à vivre. La vocation de cette jeune femme lui a été plus ou moins imposée par la vie mais si elle apprécie son travail, ses préoccupations identitaires prennent le pas sur celui-ci et la jeune femme doit se trouver une identité - ce qu'elle fait en récupérant le journal du jeune homme - pour pouvoir ensuite soigner. Shi Pingmei propose une représentation allégorique du parcours qui amène progressivement les femmes à l'autonomie : malades, puis se soignant avant de soigner. 

ses nouvelles apparaît comme témoignant d'un questionnement identitaire centré sur les origines, mises en doute, recherchées. Shi Pingmei éprouvait une grande compassion pour sa mère, seconde épouse devant élever le fils de la première épouse décédée et n'ayant de ce fait jamais eu de réelle place, du moins est-ce ainsi que Shi percevait la situation. Et on se demande dans quelle mesure ces jeunes femmes - toujours dans une quête identitaire - ne reflètent pas aussi l'histoire de cette mère reléguée à l'arrière-plan de sa propre vie. Ses rapports avec son père ont été plus faciles car s'inscrivant dans un cadre précis, celui d'un échange intellectuel au travers d'un rapport de maitre à élève. Sensible à son intelligence (elle commence à lire dès l'âge de deux ans), son père prend en charge son éducation, son fils aîné issu de son premier mariage n'étant pas doué pour les études. Cette éducation est sévère. Après l'école, Shi doit, en plus, faire les devoirs demandés par son père, parfois jusqu'à l'épuisement, mais cette exigence est aussi porteuse de la reconnaissance qu'en tant que fille, elle a eue de ce père. Un homme âgé à 
la vieillesse duquel elle sera particulièrement sensible, envisageant même de renoncer à ses études pour rester à ses côtés, ce qu'il refuse.

Dans ces nouvelles, la maladie apparait comme symbole, corps souffrant touchant d'abord la narratrice puis extérieur à celle-ci mais lié à un questionnement identitaire ou existentiel. En ce sens, la maladie est liée chez Shi Pingmei à la question de son identité qu'elle interroge dans une perspective genrée au travers de ses narratrices toujours féminines.

\section{NOTES}

1. Cf. Claude Reichler, (dir.) Le corps et ses fictions, Paris, éd. de Minuit, 1983; Brian Turner \& Zheng Yangwen (éd.), The Body in Asia, New York, Berghahn books, 2009 ; « Corps souffrants dans les littératures de la Chine et du Japon au XXe siècle », dir. par Cécile Sakaï, Gérard Siary et Victor Vuilleumier, Extrême-Orient Extrême-Occident, $\mathrm{n}^{\circ}$ 39, 2015.

2. Cf. Dominique Hoizey, Histoire de la médecine chinoise, Paris, Payot, 1988; Charlotte Furth, A Flourishing Yin: Gender in China's Medical History : 960-1665, University of California Press, 1999.

3. Parmi les nombreux ouvrages relatifs à l'influence du confucianisme sur la condition des femmes en Chine, on peut citer : Li Chenyang (éd.), The Sage and the Second Sex-Confucianism, Ethics and Gender, Chicago / La Salle, Open Court, 2000.

4. Cf. Kang-I Sun Chang, "Women's Poetic Witnessing: Late Ming and Late Qing examples », in Dynastic Crisis and Cultural Innovation - From the Late Ming to the Late Qing and Beyond, David Der-wei Wang \& Shang Wei (éd.), Cambridge (MA) / London, Harvard University Press, 2005, pp. 504-522.

5. Cf. Tani Barlow, The Question of Women in Chinese Feminism, Durham/ London, Duke University Press, 2004.

6. Cf. Grace Fong, «Writing and Illness: A Feminine Condition in Women's Poetry of the Ming and the Qing ", in The Inner Quarters and Beyond: Women Writers from Ming through Qing, Leiden / Boston, Brill, 2010, pp. 17-48.

7. Cf. Dorothy Ko, Cindirella's Sisters -A Revisionist History of Footbinding, Berkley / Los Angeles / London, University of California Press, 2005.

8. L'œuvre de Shi Pingmei ainsi que différents textes de souvenirs de ses proches ou essais lui étant consacrés ont été publiés sous la direction de Yang Yang, en trois volumes, sous le titre de Shi Pingmei zuopin ji [๔uvre de Shi Pingmei], Sanwen [Prose], ci-après désigné par SPZJ (S), Shige xiaoshuo [Poèmes et nouvelles], ci-après désigné par SPZJ (SX) et Xiju, youji shuxin [Théâtre, notes de voyages, lettres] ci-après désigné par SPZJ (XYS), Beijing, Shumu wenxian chubanshe, 1983, 1984 et 1985. En anglais, une biographie et deux textes de Shi Pingmei sont proposés par Amy D. Dooling \& Kristina M. Torgeson, Writing Women in Modern China - An Anthology of Women's Literature from the Early Twentieth Century, New York, Columbia University Press, 1998, pp. 115-134.

9. Après des publications d'étudiante, elle publie les périodiques Funü zhoubao en 1924-1925 et Qiangwei zhoubao en 1926-1928. Cf. Yang Yang, «Xinianzhe funümen he xin yidai - Tan Shi Pingmei wenxue huodong de yige tedian » [Préoccupée par les femmes et la nouvelle génération À propos d'une caractéristique de l'activité littéraire de Shi Pingmei], SPZJ (XYS), pp. 33-36. 10. Lu Jingqing, «Zhuiji Pingmei » [Souvenirs de Pingmei], in SPZJ (SX), pp. 7-13. 
11. J. Zelpha Chambers, Self, Text and Sisterhood: Shi Pingmei's Strategic deployment of Autobiographicality in The Wild Rose Weekly (Qiangwei zhoukan), Thesis (en ligne), The University of Auckland, 2015, pp. 64-93.

12. Cf. Cécile Toublet, « Le corps comme signe », Acta fabula, vol. 15, n5, «Corpora corporis », Mai 2014, URL : http://www.fabula.org/acta/document5916.php, page consultée le 23 février 2017.

13. Cf. Victor Vuilleumier, «Le corps souffrant chez Lu Xun : allégorie muette de l'obstacle et appropriation de la modernité », Extrême-Orient Extrême-Occident, $\mathrm{n}^{\circ} 39$ (2015), pp. 47-84.

14. Gu Yiyan, "Zhuiyi wode hao laoshi Shi Pingmei » [Souvenirs de mon bon professeur, Shi Pingmei], SPZJ (XYS), pp. 7-21.

15. Cf. Lu Yin, « Shi Pingmei lüezhuan » [Brève biographie de Shi Pingmei], SPZJ (XYS), p. 187.

16. Cf. Yang Yang, "Xinianzhe... ", SPJZ (XYS), pp. 29-30 ; Deng Zhuoyuan, « Pingmei nüshi de 'Zhei shi shei de zui ?' » ['De qui est-ce la faute ?' de Melle Pingmei], SPZJ (XYS), pp. 13-14 et Shi Pingmei, "Yu Zhi Shui xiansheng lun Zhuo zhu 'Zhei shi shei de zui ?' de juben - Jie yi da Deng Zhuoyuan xiansheng " [Débat avec M. Zhi Shui à propos du texte de Zhuo sur la pièce 'De qui estce la faute ?' - En guise de réponse à M. Deng Zhuoyuan], SPZJ (XYS), pp. 15-18.

17. «Bing ", p. 149.

18. Ibid., p. 152.

19. Ce journal défini comme littéraire est présent dans le roman Xiangya jiezhi 象牙戒指 [Anneaux d'ivoire, 1934], qu'il a permis de construire et dans lequel il se retrouve objet. C'est alors que la relation entre Shi Pingmei et Lu Yin prend ce caractère particulier, mêlant espace public et espace privé au travers de l'écriture : outre le journal de Lu Yin qui servira de base au roman Xiangya jiezhi, elles se mettent en scène dans leurs œuvres respectives. Et toutes deux échangent des courriers qu'elles font publier, sans se les adresser personnellement mais en les adressant aux personnages dont elles ont fait leur alter ego dans leurs nouvelles. Shi Pingmei s'adresse à Lusha (héroïne de la nouvelle « Haibin guyou » de Lu Yin), et Lu Yin lui répond sous ce nom en utilisant un des noms de plume de Shi Pingmei (Bowei), abolissant les frontières entre personne et personnage littéraire, personnage littéraire et personnalité du monde littéraire, espace privé et espace public, et, finalement, être et représentation. Cf. Nathalie Heinich, États de femmes - L'identité féminine dans la fiction occidentale, Paris, Gallimard, 1996, p. 333.

20. Gao Junyu, décédé en 1925 d'une crise d'appendicite, après que Shi Pingmei a mis fin à leur relation car il était déjà marié à une autre femme, et qu'elle refusait d'être à l'origine de leur rupture. Gao avait quand même divorcé mais Shi Pingmei était restée sur sa position. Cf. Gao Quande, «Yi Pingmei » [Souvenir de Pingmei], SPZJ (SX), pp. 14-19.

21. SPZJ (SX), pp. 207-217.

22. Cf. Kang-I Sun Chang, "Women's Poetic Witnessing: Late Ming and Late Qing examples », op. cit., pp. 504-522.

23. SPZJ (SX), pp. 164-172.

24. SPZJ (SX), pp. 184-186.

25. Cf. Sally T. Lieberman, The Mother and Narrative Politics in Modern China, Charlottesville / London, University Press of Virginia, 1998.

26. SPZJ (SX), pp. 218-233. 


\section{ABSTRACTS}

$\mathrm{Si}$, au début $\mathrm{du} \mathrm{XX}^{\mathrm{e}}$ siècle, la maladie est présente dans la vie quotidienne des écrivains et écrivaines chinois, souvent touchés par des affections comme la tuberculose, son impact sur leur écriture est peu étudié et cela bien que le ressenti individuel devienne alors l'une des sources majeures de l'écriture et que la maladie apparaisse dans leurs écrits sous une forme ou une autre. Shi Pingmei 石评梅 (1902-1928) représente un cas exemplaire, sur ce plan, tant pour des raisons biographiques que littéraires : sa première nouvelle publiée s'intitule Bing 病 [Maladie, 1923], son amant décède deux ans plus tard de maladie et elle-même succombe, trois ans après lui, des suites d'une encéphalite. Outre ce qu'elle en dit elle-même, il existe de nombreux témoignages sur son rapport à la maladie et notamment celui de son amie, l'écrivaine Lu Yin 庐隐 (1898-1934, qui relate la courte existence de Shi Pingmei, sous forme romancée, dans Xiangya jiezhi 象牙戒指 [Anneaux d'ivoire, 1934]. Shi Pingmei s'est par ailleurs battue pour faire entendre une voix qu'elle avait conscience de savoir "féminine » et c'est dans son rapport à la maladie que son discours, volontairement genré, sera analysé ici.

If, at the beginning of twentieth century, illness belongs to everyday life for Chinese writers, often affected by tuberculosis, its influence on writing is seldom studied, and this, although the individual is becoming the major source of writing and illness is often present in literary texts. Shi Pingmei's 石评梅 (1902-1928) case is particularly relevant from this angle and this, as much from a biographical point of view as from a literary one: her first short-story is entitled Bing 病 [Illness, 1923], her lover deceases two years later of illness and she dies three years after him from encephalitis. Besides what she has written herself about illness, there are many accounts about her relation to illness, like the novel written by her close friend Lu Yin 庐隐 (1898-1934), who has written a romanced biography of Shi in Xiangya jiezhi 象牙戒指 [Ivory rings, 1934]. Shi Pingmei was fighting to give audience to a voice she knew as a "feminine" one and it's in its relation to illness that her discourse - clearly gendered - is explored here.

\section{AUTHOR}

\section{JACQUELINE ESTRAN}

Maître de conférences en études chinoises, Jacqueline Estran travaille dans une perspective transculturelle sur l'histoire sociale de la littérature (Poésie et liberté dans la Chine républicaine : la revue Xinyue, 1928-1933, Harrassowitz Vlg., 2010), la condition des femmes et l'écriture féminine du XXe siècle (« Exemplaire ou à part, le destin singulier de Lin Huiyin (1904-1955) », 2012 ; « Différence, distance et prise de conscience : Su Xuelin (1897-1999) et la France », 2012 ; « Histoire d'une absence : la place du père dans les œuvres des écrivaines chinoises des années $1920 », 2014)$.

Maître de conférences in Chinese Studies, Jacqueline Estran works from a transcultural viewpoint on social history of literature (Poésie et liberté dans la Chine républicaine : la revue Xinyue, 1928-1933, Harrassowitz Vlg., 2010) and is interested in twentieth-century women's writing and related questions (" Exemplaire ou à part, le destin singulier de Lin Huiyin (1904-1955) », 2012 ; « Différence, distance et prise de conscience : Su Xuelin (1897-1999) et la France », 2012 ; 
« Histoire d'une absence : la place du père dans les œuvres des écrivaines chinoises des années $1920 », 2014)$. 